\title{
Effect of pectin feeding on obesity development and duodenal alkaline phosphatase activity in Sprague- Dawley rats fed with high-fat/high-energy diet
}

\author{
Z Šefč́íková, L Raček \\ Institute of Animal Physiology, Slovak Academy of Sciences, Košice, Slovak Republic \\ Received: September 7, 2015 \\ Accepted: May 11, 2016
}

\begin{abstract}
Purpose: The objective of this study was to evaluate whether pectin feeding would affect the small intestinal function and whether these changes would lead to obesity prevention in rats fed with high-fat diet. Three groups of weaned male rats (ad lib. fed; rats fed with diet containing 15\% w/w of citrus pectin; restrictedly pair-fed rats) were fed with either a standard diet $(9.5 \%$ fat) or a high-fat diet (30\% fat) for 10 days. Results: Our results revealed that pectin feeding led to significant decreases in body weight, energy intake and fat pad weight in rats fed with the standard as well as high-fat diet. Moreover, compared to the restrictedly pair-fed rats, in both groups of rats fed with the diet containing pectin, significant decrease in duodenal alkaline phosphatase (AP) activity was observed in histochemically stained cryostat sections. In contrast, despite their lower energy intake, restrictedly pair-fed rats showed similar fat pad deposition accompanied by unchanged values of AP activity in comparison to the controls. Conclusions: Our findings indicate that daily pectin consumption could be beneficial in suppressing body weight gain and reducing probability of obesity risk in rats fed with a high-fat diet.
\end{abstract}

Keywords: pectin, intestinal alkaline phosphatase, high-fat diet, rats, body fat

\section{Introduction}

Obesity is considered as one of the most prevalent public health problems worldwide. The main reason for obesity development is positive energy balance, i.e., the condition in which energy intake chronically exceeding energy expenditure leads to an increased fat accumulation that may impair health. Obesity is associated with metabolic diseases such as insulin resistance, diabetes, cardiovascular diseases and cancer (29). Despite growing efforts, the current strategies involved in reduction of this high prevalence of obesity have been minimally effective. A possible way in which diet composition may affect caloric intake and body weight/fat regulation is inclusion of dietary fiber in the diet (5). Pectin is one of the most important sources of dietary soluble fiber occurring as a component of plant cell walls of fruits and vegetables (28). Although pectin is not digested in the upper part of the gastrointestinal tract, its inclusion in the diet is associated with morphological and functional alterations of the small intestine as well as with changes in the physiological processes of nutrient absorption. Pectin effects are mediated through its chemical-physiological properties as it passes through the gastrointestinal tract. It has been found that addition of pectin to the diet resulted in significantly higher weight and length of intestinal tissues and also in

\footnotetext{
Corresponding author: Zuzana Šefč́iková

Institute of Animal Physiology, Slovak Academy of Sciences

Šoltésovej 4-6, 04001 Košice, Slovak Republic

Phone: +421 55 6783183; Fax: +421 55 7287842; E-mail: sefcikz@saske.sk
} 
increasing small intestinal villus height and depth of the crypts in the small and large intestines compared with rats fed with a control diet $(2,15,25)$. Data from other studies also indicate that consumption of diet with pectin leads to increased satiety, decreased food intake, body weight gain and total body fat in male rats $(1,8,24)$.

The small intestine contains several enzymes in the microvillous zone of enterocytes which play an important role in the digestion of individual food components. It has been proved that alkaline phosphatase (AP) is involved in the intestinal absorption of lipids (16). In rats, AP activity increases after fat consumption (3, 20,27); it displays circadian fluctuations closely related to food intake (19), and is evidently depressed after food deprivation (26). The impact of pectin supplementation on intestinal enzyme activity is inconsistent. Some animal studies have revealed that in pectin-fed rats the specific AP activity was significantly reduced in the upper jejunum in comparison with rats fed with basal diets (4); other results indicate that pectin stimulates ileal mucosal specific activities of AP, maltase and sucrase (6). At the present time, however, information concerning the relation between pectin feeding, intestinal enzyme activity and obesity development is lacking. In this sense, additional data may explain whether the acquired changes in small intestinal function could be potentially involved in body fat reduction and obesity prevention in weaned rats fed with high-fat diet. The purpose of this study was, therefore, to examine the effect of daily consumption of soluble dietary fiber pectin upon the growth performance and small intestinal functionality in young male rats fed with either high-fat/high-energy diet or standard laboratory diet.

\section{Materials and Methods}

\section{Animals}

Sprague-Dawley virgin rat dams (Charles River Laboratories, Prague, Czech Republic) were mated at 10 weeks of age and individually housed in plexiglass cages in a temperaturecontrolled environment of $22 \pm 2{ }^{\circ} \mathrm{C}$ with a relative humidity of $55 \pm 10 \%$ and $12 \mathrm{~h} \mathrm{light/dark}$ cycle (light on from 06:00 to 18:00) with free access to a standard laboratory diet (Laboratory diet M1, ̌̌icmanice, Czech Republic; $3.2 \mathrm{kcal} / \mathrm{g}$, with $26.3 \%$ energy as protein, $9.5 \%$ as fat, and $64.2 \%$ as carbohydrate) and tap water. Within $24 \mathrm{~h}$ of parturition, litter size was adjusted to $10 \mathrm{pups} /$ litter to ensure adequate and standardized nutrition until weaning. All litters contained males and females and were kept under the above-mentioned laboratory conditions. The weaned 30-day-old male rats were given either standard laboratory diet (experiment I) or high-fat diet (experiment II). In experiment I, the rats $(n=24)$ were randomly divided into three groups (eight animals in each group) and individually housed in plexiglass cages: (1) the control group (C) was fed with standard laboratory diet, (2) the pectin-fed group (C-P) was fed the diet prepared by combining the standard diet with $15 \%$ addition of pectin from citrus peel (Fluka No. 76280, degree of esterification 60\%), and (3) food-restricted pair-fed controls (C-PF) were food restricted to receive the average daily kcal food intake of the pectin group (C-P). Standard diet was available to them twice daily (from 8:00 and from 18:00).

In experiment II, the rats $(n=24)$ were separated into three groups (eight animals in each group) and individually housed in plexiglass cages: (1) the high-fat group (HF) in which the rats received a HF diet [catalog number 53316 (58QJ), purchased from Test Diet company (division of Lab Diet, Richmond, IN, USA); $4.04 \mathrm{kcal} / \mathrm{g}$, with $14.55 \%$ energy as protein, 30\% as fat, $55.5 \%$ as carbohydrate] from days 30 to 40 , (2) the pectin-fed group (HF-P) was fed with a HF diet supplemented with pectin from citrus peel added at 15\% level (Fluka No. 76280, 
degree of esterification 60\%), and (3) food-restricted pair-fed males (HF-PF) received the average amount of kcal consumed each day by the pectin group (HF-P). Food was divided into two doses/day (from 8:00 and from 18:00). Food intake was measured daily. On day 40, the animals were killed by decapitation without an overnight fasting (between 08:00 and 9:00), followed by removal of adipose depots and small-intestinal segments. Bilateral adipose tissue samples were collected from epididymal and perirenal regions.

\section{Enzyme Assays}

For enzyme assay, a small $(0.5 \mathrm{~cm})$ segment of the duodenum was immediately removed, the lumen rinsed in distilled water, and frozen in liquid nitrogen. Segments of the frozen tissue were cut $(8 \mu \mathrm{m})$ in the cryostat at $-22{ }^{\circ} \mathrm{C}$ and the tissue slices were transferred to glass slides and air-dried.

Analysis of AP activity was performed using a modified simultaneous azo-coupling method (18). The incubation medium contained $2.0 \mathrm{mM}$ naphthol AS-BI phosphate (Sigma, Deisenhofen, Germany), $0.8 \mathrm{mM}$ hexazotized new fuchsin (Serva, Heidelberg, Germany), $20 \mathrm{mM}$ N,N-dimethylformamide (solvent of naphthol AS-BI phosphate) and $0.05 \mathrm{M}$ veronal acetate buffer. The sections were incubated at $37{ }^{\circ} \mathrm{C}$ for $10 \mathrm{~min}$ at $\mathrm{pH} 8.9$ (21). The histochemically stained slides were visualized using image analysis and quantification of the enzyme activity (pixel intensities) was carried out in a whole section of at least four duodenal slides, and the recorded mean values were referred to one animal (22).

\section{Ethics statement}

All animal experiments were reviewed and approved by the Ethical Committee for animal experimentation of the Institute of Animal Physiology, approved by the State Veterinary and Food Administration of the Slovak Republic, and were performed in accordance with Slovak legislation (Law No. 377/2012) on the protection of animals used for experimental and other scientific purposes.

\section{Statistical analysis}

Statistical analyses were performed using the Statistica AXAZ software package (StatSoftCR, Czech Republic). Data were expressed as means \pm standard error of the mean (SEM). Statistical evaluations of the results were performed with one-way analysis of variance (ANOVA), and the post hoc Fisher's least significant difference (LSD) test was used to compare the differences between the groups. A $p<0.05$ level was considered statistically significant. Pearson's $R$ was used to assess relationships between body fat (\% b.w.) and AP activity.

\section{Results}

\section{Postweaning somatic and feeding parameters}

There were no differences in initial body weight between diet groups on day 30. On day 40, pectin-fed and restrictedly pair-fed rats showed the same final body weight and weight gain in experiment I. On the other hand, HF-P rats displayed significantly lower final body weight and lower weight gain by $12 \%$ in experiment II as compared to the HF-PF group. The foodrestricted pair-fed rats in both experiments showed lower overall body weight gain $(p<0.01)$ in comparison with the ad lib. controls (Figs 1 and 2). Pectin- and pair-fed rats consumed less 


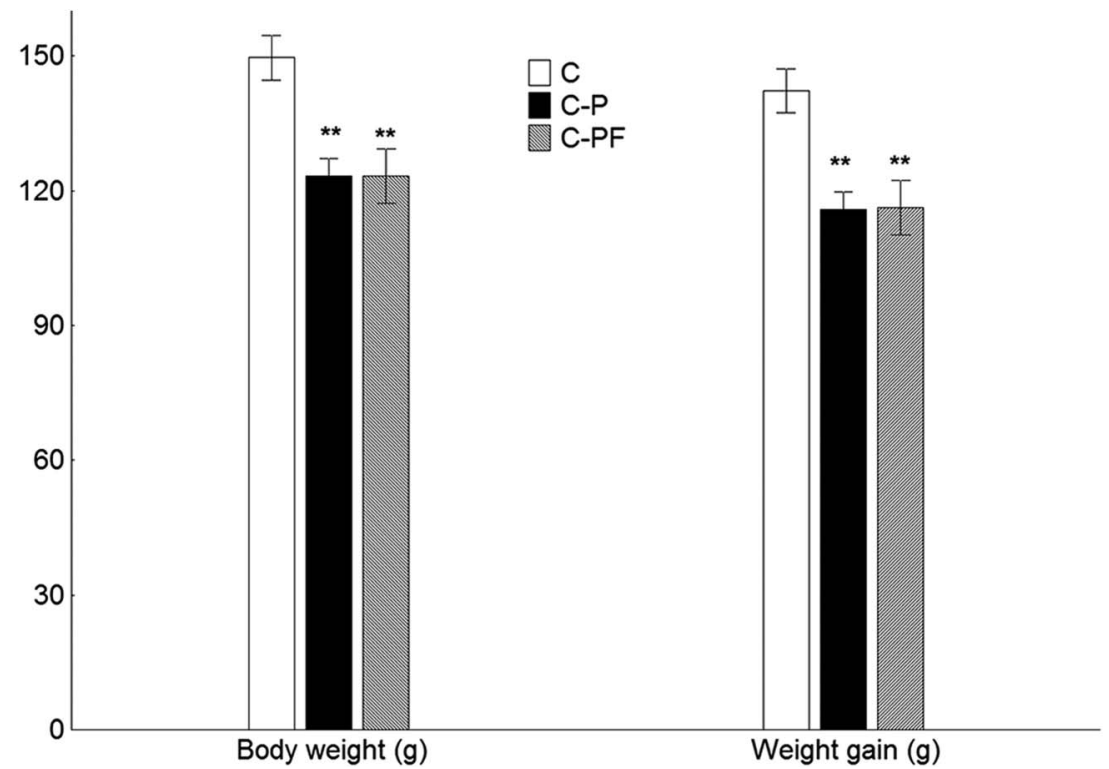

Fig. 1. Body weight (g) and weight gain (g) in 40-day-old control (C), 15\% pectin (C-P), and pair-fed (C-PF) male rats fed with standard diet. Values are means \pm SEM (eight animals/group). Significantly different from control (C) group: $* * p<0.01$ by Fisher's LSD test after ANOVA

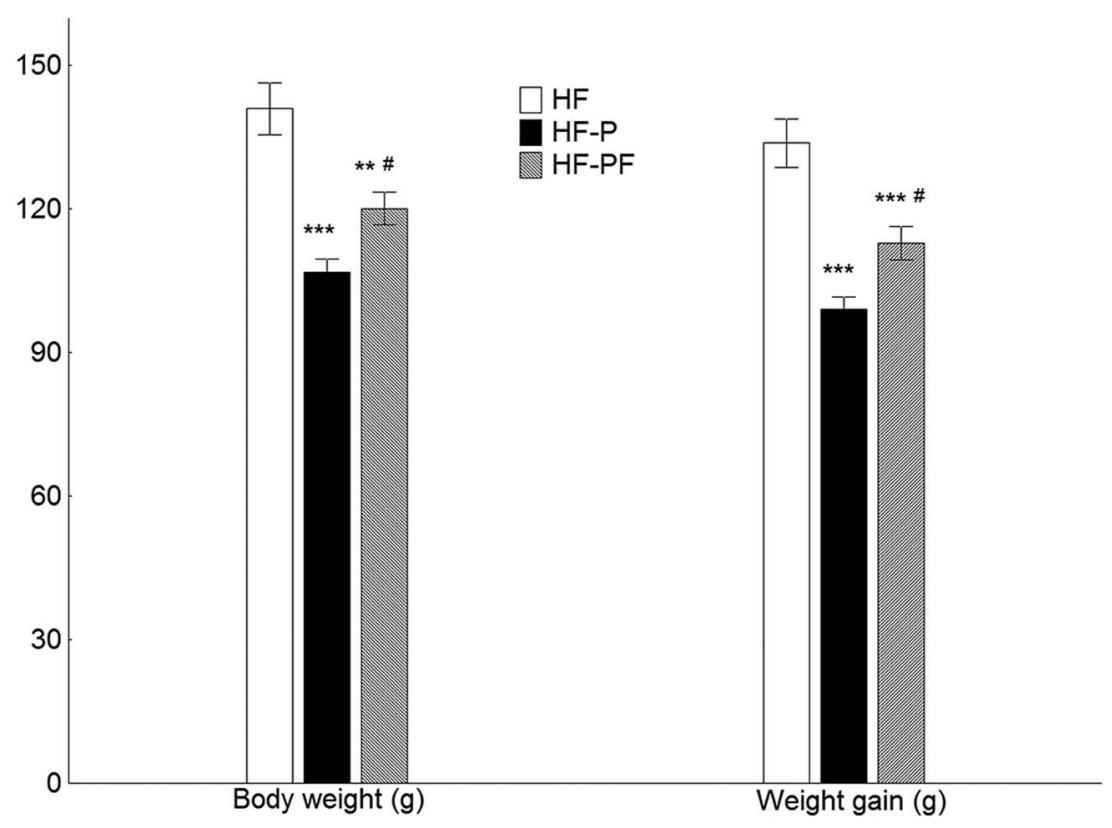

Fig. 2. Body weight (g) and weight gain (g) in 40-day-old HF, 15\% pectin (HF-P) and pair-fed (HF-PF) male rats fed with high-fat diet from days 30 to 40 . Values are means \pm SEM (eight animals/group). Significantly different from $H F$ group: ${ }^{*} p<0.01,{ }^{* *} p<0.001$ by Fisher's LSD test after ANOVA, ${ }^{*}$ significant differences between HF-P and HFPF groups 
daily energy (expressed in kcal/day) than the controls in experiments I and II. Despite their lower energy intake, food-restricted pair-fed rats revealed no significant changes in body fat pad weight in experiment I. On the other hand, rats fed with standard laboratory diet including $15 \%$ citrus pectin displayed significantly lower epididymal (about $37 \%$ ) as well as perirenal fat (about 56\%) than the pair-fed rats (Table I). Likewise, in experiment II rats fed with the high-fat diet with pectin attained a final epididymal plus perirenal fat pad weight that was significantly lower $(p<0.01)$ than in the food-restricted pair-fed animals (Table II). Pectin supplementation in the standard as well as high-fat diet resulted in significant elongation of the small intestine compared to the pair-fed groups (Tables I and II).

\section{Effects of pectin feeding on intestinal functions}

The effect of pectin supplementation on small intestinal AP activity in control, pectin- and pair-fed rats receiving the standard laboratory diet is presented in Table I. Compared to pairfed (C-PF) rats, the values of duodenal AP activity in pectin-fed rats (C-P) decreased by about $7 \%$. No alterations were observed in AP activity in rats exposed to food restriction (C-PF) compared to the controls (C). Correlation between parameters of body fat and AP activity showed significant positive correlations $(r=0.465 ; p=0.025)$ in experiment $\mathrm{I}$.

Our results also reveal that whereas the values of duodenal brush border-bound AP activity in food-restricted pair-fed (HF-PF) and HF rats remained unchanged, pectin supplementation in the high-fat diet resulted in significant duodenal AP activity reduction by $12 \%$ compared to HF-PF rats (Table II). The body fat (\%) significantly correlated with duodenal AP activity $(r=0.469 ; p=0.024)$ in experiment II.

\section{Discussion}

Overconsumption of energy-dense food and lack of physical activity leads to the growth of obesity. Obesity as a multifactorial disease results from interaction of genetic and environmental factors. Recent studies have demonstrated that obesity in rats is also closely related to disturbance of two dominant divisions of gut bacteria, especially increase in the Firmicutes and reduction of the Bacteroidetes $(11,20)$. Supplementation with apple-derived pectin in

Table I. Effect of pectin supplementation on body fat, food intake and duodenal alkaline phosphatase (AP) activity in 40-day-old male rats fed with standard diet

\begin{tabular}{|l|c|c|c|}
\hline & Control (C) & $15 \%$ pectin (C-P) & Pair-fed (C-PF) \\
\hline Epididymal fat (\% b.w.) & $0.33 \pm 0.04$ & $0.17 \pm 0.03^{* *}$ & $0.27 \pm 0.03^{\#}$ \\
\hline Perirenal fat (\% b.w.) & $0.18 \pm 0.01$ & $0.07 \pm 0.001^{* *}$ & $0.16 \pm 0.04^{\#}$ \\
\hline Food intake (kcal/day) & $44.88 \pm 1.57$ & $31.40 \pm 1.39^{* * *}$ & $35.79 \pm 1.65^{* * *}$ \\
\hline Small intestine (cm) & $110.00 \pm 2.30$ & $121.88 \pm 2.49^{* *}$ & $104.13 \pm 2.22^{\#}$ \\
\hline AP activity & $12.91 \pm 0.17$ & $11.95 \pm 0.31^{*}$ & $12.91 \pm 0.22^{\#}$ \\
\hline
\end{tabular}

Values are means \pm SEM (eight animals/group). AP activity is given as density values (pixel intensities) in duodenal enterocytes at a wavelength of $520 \mathrm{~nm}$. Significantly different from control (C) group: *p<0.05, $* * p<0.01, * * * p<0.001$ by Fisher's LSD test after ANOVA, ${ }^{*}$ significant differences between C-P and C-PF groups 
Table II. Effect of pectin supplementation on body fat, food intake and duodenal AP activity in 40-day-old male rats fed with high-fat diet from days 30 to 40

\begin{tabular}{|l|c|c|c|}
\hline & HF & $15 \%$ pectin (HF-P) & Pair-fed (HF-PF) \\
\hline Epididymal fat (\% b.w.) & $0.37 \pm 0.04$ & $0.15 \pm 0.03^{* * *}$ & $0.33 \pm 0.02^{\#}$ \\
\hline Perirenal fat (\% b.w.) & $0.32 \pm 0.03$ & $0.12 \pm 0.01^{* * *}$ & $0.32 \pm 0.04^{\#}$ \\
\hline Food intake (kcal/day) & $50.12 \pm 1.53$ & $36.06 \pm 2.90^{* * *}$ & $40.63 \pm 1.31^{* *}$ \\
\hline Small intestine (cm) & $104.50 \pm 2.08$ & $110.63 \pm 1.08^{*}$ & $102.88 \pm 1.37^{\#}$ \\
\hline AP activity & $15.81 \pm 0.42$ & $13.46 \pm 0.31 * * *$ & $15.29 \pm 0.31^{\#}$ \\
\hline
\end{tabular}

Values are means \pm SEM (eight animals/group). AP activity is given as density values (pixel intensities) in duodenal enterocytes at wavelength of $520 \mathrm{~nm}$. Significantly different from HF group: ${ }^{*} p<0.05, * * p<0.01, * * * p<0.001$ by Fisher's LSD test after ANOVA, " significant differences between HF-P and HF-PF groups

obese rats fed with high-fat diet restored bacteria levels to normal ranges (11). Pectin addition alleviated metabolic endotoxemia and inflammation in obese rats (11).

This study demonstrates how pectin addition to diets with two different fat contents influences body weight, adiposity and small intestinal enzyme activity in young male rats. Our present results indicate that reduced caloric intake in pectin-fed rats leads to significantly decreased body fat content in comparison with the pair-fed group, which is in agreement with previous findings suggesting similarly decreased body fat accumulation in rats fed with a diet containing pectin $(1,2,8)$. Decreased overall food intake in pectin-fed rats was accompanied with increased satiety and with increased circulating concentrations of the gut satiety hormones (1). In contrast, several studies have suggested that pectin does not influence food intake and growth rate $(7,9)$.

It is known that the gastrointestinal tract plays an important role in the physiological process of digestion and absorption. It has been proved that addition of pectin to the diet decreases intestinal glucose (14) and fat absorption (23) in these rats. Moreover, regarding the role of intestinal enzymes, AP is considered as a brush border-bound representative enzyme that has a broad range of roles, involving regulation of lipid absorption (16). Its activity increased markedly after fat feeding $(13,17,20)$ and decreased after fasting, while refeeding elevated it to the pre-fasting level (26). AP in rodents is present at its highest level in the duodenum, and its activity decreases gradually toward the jejunum and ileum (10). The presented results show that besides the reduction of adiposity, consumption of a standard diet with citrus pectin also affected the small intestinal function, i.e., significantly decreased duodenal AP activity was found in 40-day-old pectin-fed rats in comparison with the pair-fed group. This is in line with previous studies showing significantly reduced small intestinal AP activity after addition of soluble dietary fibers (pectin and guar gum) to the rat diet $(4,12)$. In contrast, another rat study using dietary pectin reported elevated ileal specific AP activity (6). At the present time, the available data on the impact of pectin on somatic, feeding and intestinal parameters are in some cases controversial. These contradictory data may result from different types of pectin (chemical and physical properties) as well as from various experimental periods and methods used in the experiments.

The restrictedly pair-fed rats reduced their weight gain without substantial changes in body fat pads or in enzyme activity. Our results concerning decreased AP activity in rats fed 
with pectin compared to pair-fed rats, when both pectin- and pair-fed groups displayed lower energy intake, clearly indicate that these enzymatic changes developed as a consequence of pectin feeding, and were not dependent on decreased energy intake. Moreover, exposure of rats to diet with $30 \%$ energy as fat after weaning resulted in similar responsiveness in growth and feeding parameters as recorded in rats fed a diet with $9.5 \%$ energy as fat, i.e., it significantly decreased adiposity (epididymal and perirenal fat pad weight) and food consumption in HF-P rats was observed. In addition, our results indicate that except modified somatic and feeding parameters, significantly reduced AP activity was found as well. The exact mechanism by which pectin feeding may lead to duodenal changes has not been fully elucidated. Further work is needed to obtain more findings about the benefit of pectin inclusion in diet.

\section{Conclusions}

The results of our study show that postweaning daily inclusion of soluble dietary fiber (pectin) into diets with two different fat contents led to decreases in food intake, weight gain and fat pad weight in male rats. It seems that citrus pectin is an important factor against obesity development, and this effect might be partially explained by the significant reduction of intestinal AP activity. Additional studies focusing on enzymatic plasticity might be helpful for future pharmacological and dietary strategies for prevention of obesity.

\section{Acknowledgements}

The authors thank Andrew Billingham for his revision of the English text and Dr. Štefan Mozeš for his useful comments. This study was supported by VEGA grant 2/0011/14 of the Slovak Academy of Sciences.

\section{Conflict of interest}

The authors declare no conflict of interest.

\section{REFERENCES}

1. Adam CL, Williams PA, Dalby MJ, Garden K, Thomson LM, Richardson AJ, Gratz SW, Ross AW: Different types of soluble fermentable dietary fibre decrease food intake, body weight gain and adiposity in young adult male rats. Nutr. Metab. (Lond.) 11, 36 (2014)

2. Adam CL, Williams PA, Garden KE, Thomson LM, Ross AW: Dose-dependent effects of a soluble dietary fibre (pectin) on food intake, adiposity, gut hypertrophy and gut satiety hormone secretion in rats. PLoS ONE 10, e0115438 (2015)

3. Almeida FN, Andrade ML, Franzói de Moraes SM, Chimin P, de Almeida KN, Peralta RM, Natali MR: Obese adult phenotype. Adaptations of small intestine to cafeteria diet and aerobic physical training after weaning. Sci. Sports 29, 20-26 (2014)

4. Brown RC, Kelleher J, Losowsky MS: The effect of pectin on the structure and function of the rat small intestine. Br. J. Nutr. 42, 357-365 (1979)

5. Burton-Freeman B: Dietary fiber and energy regulation. J. Nutr. 130 (2S Suppl.), 272S-275S (2000)

6. Chun W, Bamba T, Hosoda S: Effect of pectin, a soluble dietary fiber, on functional and morphological parameters of the small intestine in rats. Digestion 42, 22-29 (1989)

7. Dongowski G, Lorenz A, Proll J: The degree of methylation influences the degradation of pectin in the intestinal tract of rats and in vitro. J. Nutr. 132, 1935-1944 (2002)

8. Fåk F, Jakobsdottir G, Kulcinskaja E, Marungruang N, Matziouridou C, Nilsson U, Stålbrand H, Nyman M: The physico-chemical properties of dietary fibre determine metabolic responses, short-chain fatty acid profiles and gut microbiota composition in rats fed low- and high-fat diets. PLoS ONE 10 (5), e0127252 (2015) 
9. Henningsson AM, Björck IM, Nyman EM: Combinations of indigestible carbohydrates affect short-chain fatty acid formation in the hindgut of rats. J. Nutr. 132, 3098-3104 (2002)

10. Jang I, Jung K, Cho J: Influence of age on duodenal brush border membrane and specific activities of brush border membrane enzymes in Wistar rats. Exp. Anim. 49, 281-287 (2000)

11. Jiang T, Gao X, Wu C, Tian F, Lei Q, Bi J, Xie B, Wang HY, Chen S, Wang X: Apple-derived pectin modulates gut microbiota, improves gut barrier function, and attenuates metabolic endotoxemia in rats with diet-induced obesity. Nutrients 8, 126 (2016)

12. Johnson IT, Gee JM, Mahoney RR: Effect of dietary supplements of guar gum and cellulose on intestinal cell proliferation, enzyme levels and sugar transport in the rat. Br. J. Nutr. 52, 477-487 (1984)

13. Kaur J, Madan S, Hamid A, Singla A, Mahmood A: Intestinal alkaline phosphatase secretion in oil-fed rats. Dig. Dis. Sci. 52, 665-670 (2007)

14. Kim M: High-methoxyl pectin has greater enhancing effect on glucose uptake in intestinal perfused rats. Nutrition 21, 372-377 (2005)

15. Knapp BK, Bauer LL, Swanson KS, Tappenden KA, Fahey GC Jr, de Godoy MR: Soluble fiber dextrin and soluble corn fiber supplementation modify indices of health in cecum and colon of Sprague-Dawley rats. Nutrients 5, 396-410 (2013)

16. Lalles JP: Intestinal alkaline phosphatase: multiple biological roles in maintenance of intestinal homeostasis and modulation by diet. Nutr. Rev. 68, 323-332 (2010)

17. Lallès JP, Orozco-Solís R, Bolaños-Jiménez F, de Coppet P, Le Dréan G, Segain JP: Perinatal undernutrition alters intestinal alkaline phosphatase and its main transcription factors KLF4 and Cdx1 in adult offspring fed a high-fat diet. J. Nutr. Biochem. 23, 1490-1497 (2012)

18. Lojda Z, Gossrau R, Schibler TH (1979): Enzyme Histochemistry: A Laboratory Manual. Springer-Verlag, Berlin

19. Martinková A, Lenhardt L, Mozeš Š: Effect of neonatal MSG treatment on day-night alkaline phosphatase activity in the rat duodenum. Physiol. Res. 49, 339-345 (2000)

20. Mozeš Š, Bujňáková D, Šefćíková Z, Kmet V: Developmental changes of gut microflora and enzyme activity in rat pups exposed to fat-rich diet. Obesity 16, 2610-2615 (2008)

21. Mozeš Š, Lenhardt L, Martinková A: A quantitative histochemical study of alkaline phosphatase activity in isolated rat duodenal epithelial cells. Histochem. J. 30, 1-7 (1998)

22. Mozeš Š, Šef̌́íková Z, Lenhardt L: Functional changes of the small intestine in over- and undernourished suckling rats support the development of obesity risk on a high-energy diet in later life. Physiol. Res. 56, 183192 (2007)

23. Munakata A, Iwane S, Todate M, Nakaji S, Sugawara K: Effects of dietary fiber on gastrointestinal transit time, fecal properties and fat absorption in rats. Tohoku J. Exp. Med. 176, 227-238 (1995)

24. Pirman T, Patureau Mirand P, Orešnik A, Salobir J: Effects of dietary pectin on protein digestion and metabolism in growing rats. Acta Agric. Slov. 94, 111-119 (2009)

25. Pirman T, Ribeyre MC, Mosoni L, Remond D, Vrecl M, Salobir J, Patureau Mirand P: Dietary pectin stimulates protein metabolism in the digestive tract. Nutrition 23, 69-75 (2007)

26. Raček L, Lenhardt L, Mozeš Š : Effect of fasting and refeeding on duodenal alkaline phosphatase activity in monosodium glutamate obese rats. Physiol. Res. 50, 365-372 (2001)

27. Scoaris CR, Rizo GV, Roldi LP, de Moraes SM, de Proença AR, Peralta RM, Natali MR: Effects of cafeteria diet on the jejunum in sedentary and physically trained rats. Nutrition 26, 312-320 (2010)

28. Thakur BR, Singh RK, Handa AK: Chemistry and uses of pectin - a review. Crit. Rev. Food Sci. Nutr. 37 , 47-73 (1997)

29. World Health Organization: Obesity and overweight; Fact sheet $N^{\circ} 311$. http://www.who.int/mediacentre/ factsheets/fs311/en/. Updated Jan 2015 